NASA Contractor Report 3389

\title{
A Three-Dimensional Turbulent Compressible Subsonic Duct Flow Analysis for Use With Constructed Coordinate Systems
}

R. Levy, H. McDonald, W. R. Briley, and J. P. Kreskovsky 
NASA Contractor Report 3389

\section{A Three-Dimensional Turbulent Compressible Subsonic Duct Flow Analysis for Use With Constructed Coordinate Systems}

R. Levy, H. McDonald, W. R. Briley, and J. P. Kreskovsky

Scientific Research Associates, Inc. Glastonbury, Connecticut

Prepared for

Lewis Research Center under Contract NAS3-21735

\section{N/Sก}

National Aeronautics

and Space Administration

Scientific and Technical Information Branch

1981 
TABLE OF CONTENTS

Page

SUMMARY . . . . . . . . . . . . . . . . . . . . . . . . . . . . 1 INTRODUCTION . . . . . . . . . . . . . . . . . . . . . . . . 2 ANALYSIS . . . . . . . . . . . . . . . . . . . . . . . . . 3

Coordinate Systems . . . . . . . . . . . . . . . . . . . . 3

Governing Equations . . . . . . . . . . . . . . . . . . . . . . 5

Flow in Ducts with Superelliptic Cross Sections . . . . . . . . . . . . . 9

Potential flow . . . . . . . . . . . . . . . . . . . . . . . 13

Calculations. . . . . . . . . . . . . . . . . . . . . . . . . 14

SUMMARY AND CONCLUSIONS . . . . . . . . . . . . . . . . . . . . . 16

REFERENCES . . . . . . . . . . . . . . . . . . . . . . . . . 17

FIGURES . . . . . . . . . . . . . . . . . . . . . . . . . . . . . . . 18

SYMBOL TABLE . . . . . . . . . . . . . . . . . . . . . . . . . 28

APPENDIX - COMPUTER CODE INPUT AND OUTPUT . . . . . . . . . . . . . . . . . . 29 


\section{SUMMARY}

An approximate analysis is presented for computation of three-dimensional subsonic flow in straight and curved diffusers. The development parallels that of Briley and McDonald for forward-marching solution of viscous primary and secondary flows, but differs in the coordinate formulation used and in details of the approximations. The present formulation is intended to facilitate the use of constructed coordinates in circumstances where it is difficult to maintain smooth behavior in higher derivatives. This analysis is applicable to nonorthogonal coordinate systems having a curved centerline and planar transverse coordinate surfaces normal to the centerline. The primary flow direction is taken to coincide with the local direction of the duct centerline and is hence normal to transverse coordinate planes. The formulation utilizes vector components (velocity, vorticity, transport equations) defined in terms of local Cartesian directions aligned with the centerline tangent, although the governing equations themselves are expressed in general nonorthogonal courdinates. For curved centerlines, these vector quantities are redefined in new local Cartesian directions at each streamwise location. The use of local Cartesian variables and fluxes leads to governing equations which require only first derivatives of the coordinate transformation, and this provides for the aforementioned ease in using constructed coordinates. The analysis is applied to a particular family of duct and diffuser genteries having curved centerlines and superelliptic cross sections, using a nonorthogonal constructed coordinate system. Present computed results are compared with available experimental measurements for different but related flow conditions. Qualitative agreement is observed with regard to the formation of secondary flows, migration of streamwise vortices, and distortion of the primary flow. Additional experimental data is needed to provide a basis for detailed quantitative evaluation of the analysis and flow predictions. 


\section{INTRODUCTION}

A continuing problem in the design of inlets for airbreathing propulsion systems is the design of efficient subsonic diffusers. In the case of three-dimensional inlet diffusers, the cross-sectional shape of the ducting varies in the axial direction and it is frequently necessary to introduce offset bends (curved duct centerlines). The complicated diffuser geometry and the offset bends introduce strong secondary flows which have important effects on both diffuser performance and engine/inlet compatibility. A generalized subsonic diffuser analysis capable of being used as a design tool must account for several physical phenomena which frequently occur in practical diffusers. First, the analysis must be capable of treating the case when the wall boundary layers are turbulent and when the thickness is comparable to the local duct radius. Secondly, the analysis must be capable of treating the strong secondary flows which are commonly encountered in threedimensional diffusers.

Although solution of the three-dimensional Navier-Stokes equations would be definitive, more cost effective approximate methods are proving attractive. One such approximate method has been developed by Briley and McDonald (Ref. 1) where a set of governing equations are developed by introducing approximations into the Navier-Stokes equations and then solved by spatial marching. This paper reports on a study to modify the analysis of Ref. 1 for use in constructed coordinate systems which may be nonorthogonal. By a judicious choice of dependent variables and of forms of the equations, some of the smoothness requirements for the coordinate system have been relaxed. 
ANALYSIS

\section{Coordinate Systems}

A coordinate system is more than merely the grid points at which the governing equations are written. The coordinate system manifests itself as the geometric terms in the governing equations as is related to resolution of the physical processes and various length scales within the problem being studied. Although it is easy to overlook the part that the coordinate system plays in the governing equations, the requirements for a "good" coordinate system are found by finding the role these geometric terms play in the fluid dynamic equations. Different forms of the Navier-Stokes equations can impose different requirements for a coordinate system. For illustrative purposes a viscous term from two forms of the two-dimensional Navier-Stokes equations will be used.

First consider a point $P$ in reference Cartesian coordinates $\left(x^{1}, x^{2}\right)$. The generalized coordinate lines are $\mathrm{y}^{1}$ and $\mathrm{y}^{2}$ passing through $\mathrm{P}$ and the Cartesian velocities are $u^{1}$ and $u^{2}$. Using the quasi-linear form of the Navier-Stokes equations and Cartesian velocity components one of the viscous terms in the $x^{1}$ momentum equation would be

$$
\frac{J}{\operatorname{Re}} \frac{\partial Y^{2}}{\partial X^{2}} \frac{\partial}{\partial Y^{2}} \quad\left(\mu \frac{\partial Y^{2}}{\partial X^{2}} \frac{\partial u^{\prime}}{\partial Y^{2}}\right)
$$

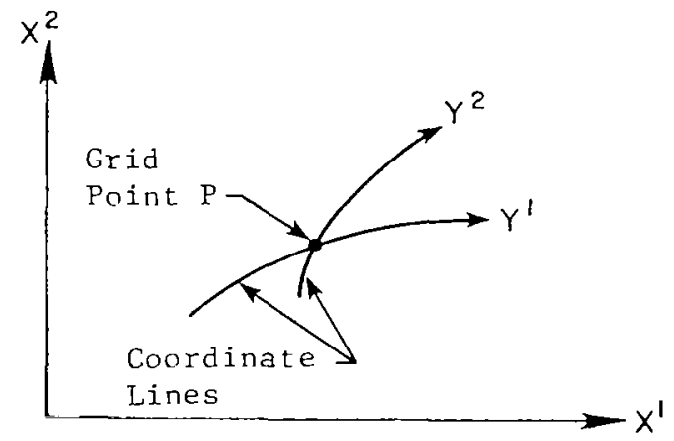

where $J$ is the Jacobian of the transformation from $x^{i}$ into $y^{i}$ and $\partial y^{2} / \partial x^{2}$ is a component of the inverse transformation tensor. The Jacobian as well as the elements of the inverse transformation tensor are all related to the gradient of the position vector at the computational grid points relative to a fixed, reference Cartesian coordinate system. 
Before continuing, let us identify this term in familiar Cartesian coordinates. In this case $J=1, \partial y^{2} / \partial x^{2}=1, y^{2}=x^{2}$ and $u^{1}=U$. By performing these simplifications we get:

$$
\frac{1}{\operatorname{Re}} \cdot 1 \cdot \frac{\partial}{\partial Y}\left(\mu \cdot 1 \cdot \frac{\partial u}{\partial Y}\right)
$$

or

$$
\frac{1}{R e} \frac{\partial}{\partial Y}\left(\mu \frac{\partial u}{\partial Y}\right)
$$

which is the familiar form of the dominant viscous term in the Cartesian $x$ momentum equation. From the simplicity of (2) one could easily forget the complexity of the term when expressed in general nonorthogonal form; but the complexity is real and the geometric terms are present and must be properly treated.

Returning to (1) it can be seen that the mathematical requirements on the inverse transformation tensor are the same as those on the fluids variables. That is, each element of (1) must be continuous, have continuous derivatives and be well defined by the computational grid points being used to solve the problem. Since the gcomctric terms in (1) are directly related to first derivatives of the locations of the computational grid points and these are in turn differentiated, the requirement on both the geometry and the velocity field is that in going from one grid point to another the second derivatives must be continuous and well defined. In addition, if the discretization of the differential equations into the difference equations is to be accurate, the geometric derivatives must not be large compared to the fluid dynamic terms.

As a second example, a corresponding viscous term is now examined in the general orthogonal form of the Navier-Stokes equations. For coordinate lines $\mathrm{y}^{1}$ and $\mathrm{y}^{2}$ and for velocity components along the coordinate directions $u_{1}$ and $u_{2}$ this viscous terms is

$$
\frac{1}{\operatorname{Re} h_{1} h_{2}} \frac{\partial}{\partial Y^{2}}\left(\begin{array}{ll}
\frac{h_{1}{ }^{2} \mu}{h_{2}} & \frac{\partial \frac{u_{1}}{h_{1}}}{\partial Y^{2}}
\end{array}\right)
$$

where $h_{1}$ and $h_{2}$ are the metrics (e.g.)

$$
h_{1}=\left(\left(\frac{\partial x^{\prime}}{\partial y^{\prime}}\right)^{2}+\left(\frac{\partial x^{2}}{\partial y^{\prime}}\right)^{2}\right)^{1 / 2}
$$

To verify that this viscous term corresponds to the term examined in ( 1 ) for the case of Cartesian coordinates the metrics are all set to unity, $u_{1}=U, x^{1}=y^{1}, x^{2}=y^{2}$ : 


$$
\frac{1}{R \theta} \frac{\partial}{\partial Y^{2}}\left(\mu \frac{\partial u}{\partial Y^{2}}\right)
$$

which corresponds to (2).

Examination of (3a) shows that there is a second derivative of $h_{1}$ Implying that $h_{1}$ must be continuous and have continuous derivatives. Equation ( $3 b$ ) shows that $h_{1}$ is itself a first derivative of the coordinate system. Thus, the requirement on the coordinate system for general orthogonal systems is that the coordinates must have continuous and well defined third derivatives.

In constructing coordinate systems one of the constraints is the required smoothness. One measure of the required smoothness is the order of continuous derivatives required. The above example demonstrates that the form of the governing equations can directly influence the adequacy of the coordinate system. This observation played a major role in both the selection of the forms of the governing equations and in the modifications made to the analysis of Ref. 1, which will now be discussed.

\section{Governing Equations}

The equations used in this analysis are based on the steady compressible Navier-Stokes equations. Three basic assumptions are made to permit a forward marching solution of the subsonic flow problem. First, the flow can be analyzed as being a primary flow with a transverse secondary flow. This primary flow is assumed to be approximately aligned with one of the coordinate directions. Second, the pressure field can be represented by a three-dimensional a priori known pressure field, such as comes from a three-dimensional potential flow analysis, plus a onedimensional correction for viscous blockage effects and pressure loss.

$$
P=P_{3-0}\left(x^{1}, x^{2}, x^{3}\right)+P_{1-D}\left(x^{3}\right)
$$

The three-dimensional pressure field contains elliptic information about the flow (upstream influence). Third, the assumption of negligible second derivatives in the marching direction is also required to create an initial boundary value problem which can be solved by forward marching in one of the coordinate directions.

With these assumptions the Navier-Stokes equations are written using Cartesian velocities, $u_{i}$, and Cartesian coordinates, $x^{i}$. Although subsequent forms of these equations are to be evaluated in general nonorthogonal coordinates, $y^{i}$, the use of 
Cartesian velocities and Cartesian directions for the vector equations will result in the favorable smoothness properties discussed above. The Cartesian coordinate system $x^{i}$, is rotated at each computational step to keep the $x^{3}$ Cartesian direction aligned with the tangent to the duct centerline ensuring that even when the duct contains large bends, the $x^{3}$ coordinate direction is always roughly aligned with the primary flow direction. The $\mathrm{x}^{1}$ coordinate direction is aligned with the outward facing normal. Special treatment of cases where the centerline curvature is zero is readily provided. The vector quantities, velocity and vorticity, are also transformed to stay consistent with the Cartesian directions.

The velocity vector, $\mathrm{V}$, is decomposed into a primary flow component, $\mathrm{u}_{3}$, normal to the cross plane and a secondary flow component. The secondary flow is further decomposed into parts associated with the irrotational cross flow, $\bar{V}_{\phi}$, and the rotational cross flow, $\overline{\mathrm{V}}_{\psi}$ :

$$
\begin{gathered}
\bar{v}=\bar{u}_{3}+\bar{v}_{s} \\
\bar{v}=\bar{u}_{3}+\bar{v}_{\phi}+\bar{v}_{\psi}
\end{gathered}
$$

where

$$
\begin{aligned}
& \nabla_{s} \times\left(\bar{v}_{\phi}\right)=0 \\
& \nabla_{s} \cdot\left(\rho \bar{v}_{\psi}\right)=0
\end{aligned}
$$

and $\nabla_{S}$ is the surface gradient operator in the cross plane:

$$
\nabla_{s}=\frac{\partial}{\partial X^{1}} \bar{i}+\frac{\partial}{\partial X^{2}} \bar{j}=\frac{\partial Y^{n}}{\partial X^{l}} \frac{\partial}{\partial Y^{n}} \bar{i}+\frac{\partial Y^{n}}{\partial X^{2}} \frac{\partial}{\partial Y^{n}} \bar{j}
$$

for $n=1,2$.

Since the $x^{3}$ direction is the primary flow direction, the $x^{3}$ momentum equation in Cartesian coordinates is

$$
\begin{gathered}
\rho u_{j} \frac{\partial u_{3}}{\partial x^{j}}=-\frac{\partial P_{3}-D}{\partial x^{3}}-\frac{\partial P_{1}-D}{\partial x^{3}}+F_{3} \\
+\frac{\partial}{\partial x^{j}}\left[\mu_{e}\left(\left(1-\delta_{j 3}\right)\left(\frac{\partial u_{3}}{\partial x^{j}}+\frac{\partial u_{j}}{\partial x^{3}}\right)-\frac{2}{3} \delta_{3 j}\left(1-\delta_{k} 3\right) \frac{\partial u_{k}}{\partial x^{k}}\right)\right]
\end{gathered}
$$


where $\mathrm{F}_{3}$ is the body force in the $\mathrm{x}^{3}$ direction and $\mu_{e}$ is the effective viscosity. Using the nonorthogonal computational coordinates, $y^{i}$, the $x^{3}$ momentum equation:

$$
\begin{aligned}
\rho u_{j} \frac{\partial Y^{i}}{\partial x^{j}} \frac{\partial u_{3}}{\partial Y^{i}}= & -\frac{\partial Y^{i}}{\partial X^{3}} \frac{\partial P_{3}-D}{\partial Y^{i}}-\frac{\partial Y^{3}}{\partial x^{3}} \frac{\partial P_{1-D}}{\partial Y^{3}}+F_{3} \\
+ & \frac{\partial Y^{i}}{\partial x^{j}} \frac{\partial}{\partial Y^{i}}\left[\mu _ { e } \left(\left(1-\delta_{j} 3\right)\left(\frac{\partial Y^{n}}{\partial x^{j}} \frac{\partial u_{3}}{\partial Y^{n}}+\frac{\partial Y^{n}}{\partial x^{3}} \frac{\partial u_{j}}{\partial Y^{n}}\right)\right.\right. \\
& \left.\left.-\frac{2}{3} \delta_{3 j}\left(1-\delta_{k}\right) \frac{\partial Y^{n}}{\partial x^{k}} \frac{\partial u_{k}}{\partial Y^{n}}\right)\right]
\end{aligned}
$$

is solved along with the global mass flux conservation relation

$$
\iint \frac{\partial p u_{3}}{\partial x^{3}} d x^{1} d x^{2}=0
$$

the energy equation:

$$
h_{0}=\text { const }
$$

and the equation of state:

$$
P=P_{3-0}+P_{1-D}=\rho R T
$$

to determine $u_{3}, \rho$, and $P_{1-D}\left(x^{3}\right)$.

The continuity equation is written as:

$$
\nabla \cdot(\rho \bar{v})=\frac{\partial u_{3} \rho}{\partial x^{3}}+\nabla_{s} \cdot\left(\rho \bar{v}_{\phi}\right)=0
$$

Introducing a potential function $\phi$ :

$$
\nabla_{s} \cdot\left(\rho \nabla_{s} \phi\right)=-\frac{\partial u_{3} \rho}{\partial x^{3}}
$$

this Poisson equation is solved in the form:

$$
\begin{aligned}
\frac{\partial Y^{j}}{\partial X^{i}} \frac{\partial}{\partial Y^{j}}\left(\rho \frac{\partial Y^{k}}{\partial X^{i}}\right) & \frac{\partial \phi}{\partial Y^{k}}+\rho\left(\frac{\partial Y^{k}}{\partial X^{i}}\right)^{2} \frac{\partial^{2} \phi}{\partial Y^{2}} \\
& +2 \rho \frac{\partial Y^{i}}{\partial X^{i}} \frac{\partial Y^{2}}{\partial X^{i}} \frac{\partial^{2} \phi}{\partial Y^{l} \partial Y^{2}}=-\frac{\partial Y^{n}}{\partial X^{3}} \frac{\partial \rho u_{3}}{\partial Y^{n}}
\end{aligned}
$$


for $k=1,2$ where $\phi$ is defined by:

$$
\nabla_{s} \phi=\bar{v}_{\phi}
$$

and satisfies ( 8 ).

The remaining unknown is the rotational cross flow velocity component, $\bar{v}_{\psi}$. This is found by cross differentiating and combining $x^{1}$ and $x^{2}$ momentum equations to form the vorticity transport equation in the $x^{3}$ direction. One form of this equation is:

$$
\begin{aligned}
& u_{j} \frac{\partial \Omega_{3}}{\partial x^{j}}-\Omega_{j} \frac{\partial u_{3}}{\partial x^{j}}-u_{j} \frac{\partial \rho}{\partial x^{j}} \frac{\Omega_{3}}{\rho}=\frac{\mu_{e}}{\rho} \frac{\partial^{2} \Omega_{3}}{\partial x^{j 2}}+\frac{\partial\left(F_{1} / \rho\right)}{\partial x^{2}}-\frac{\partial\left(F_{2} / \rho\right)}{\partial x^{1}} \\
& +\frac{1}{\rho}\left(\frac{\partial \mu_{e}}{\partial x^{2}}-\frac{\partial \mu_{e}}{\partial x^{1}}\right) \frac{\partial}{\partial x^{\prime}}\left(\left(1-\delta_{3 j}\right)\left(\frac{\partial u_{1}}{\partial x^{j}}-\frac{\partial u_{2}}{\partial x^{j}}\right)+\frac{\partial u_{j}}{\partial x^{1}}-\frac{\partial u_{j}}{\partial x^{2}}-\frac{2}{3}\left(\delta_{1 j}-\delta_{2 j}\right) \frac{\partial u_{k}}{\partial x^{k}}\right) \\
& +\frac{1}{\rho} \frac{\partial \mu_{e}}{\partial x^{j}}\left(\frac{\partial}{\partial x^{2}}\left(\frac{\partial u_{1}}{\partial x^{j}}-\frac{2}{3} \delta_{1 j} \frac{\partial u_{k}}{\partial x^{k}}\right)-\frac{\partial}{\partial x^{1}}\left(\frac{\partial u_{2}}{\partial x^{j}}-\frac{2}{3} \delta_{2 j} \frac{\partial u_{k}}{\partial x^{k}}\right)\right) \\
& +\frac{1}{\rho} \frac{\partial^{2} \mu_{e}}{\partial x^{2} \partial x^{j}}\left(\frac{\partial u_{1}}{\partial x^{j}}+\frac{\partial u j}{\partial x^{1}}-\frac{2}{3} \delta_{1 j} \frac{\partial u_{k}}{\partial x^{k}}\right) \\
& +\frac{1}{\rho} \frac{\partial^{2} \mu_{e}}{\partial x^{1} \partial x^{j}}\left(\frac{\partial u_{2}}{\partial x^{j}}+\frac{\partial u_{j}}{\partial x^{2}}-\frac{2}{3} \delta_{2 j} \frac{\partial u_{k}}{\partial x^{k}}\right) \\
& +\frac{1}{\rho^{2}}\left(\frac{\partial \rho}{\partial x^{2}} \frac{\partial P_{3-D}}{\partial x^{1}}-\frac{\partial \rho}{\partial x^{1}} \frac{\partial P_{3-D}}{\partial x^{2}}\right)
\end{aligned}
$$

where $u_{1}$ and $u_{2}$ are components of

$$
u_{1} \bar{i}+u_{2} \bar{j}=\bar{v}_{s}=\bar{v}_{\phi}+\bar{v}_{\psi}
$$

8 
and the vorticity is defined as:

$$
\begin{aligned}
& \Omega_{1}=\frac{\partial u_{2}}{\partial x^{3}}-\frac{\partial u_{3}}{\partial x^{2}} \\
& \Omega_{2}=\frac{\partial u_{3}}{\partial x^{1}}-\frac{\partial u_{1}}{\partial x^{3}} \\
& \Omega_{3}=\frac{\partial u_{1}}{\partial x^{2}}-\frac{\partial u_{2}}{\partial x^{1}}
\end{aligned}
$$

To solve this equation for $\bar{v}_{\psi}$ a stream function, $\bar{\psi}$ in the $x^{3}$ direction, is introduced satisfying:

$$
\nabla_{\mathrm{S}} \times \bar{\psi}=\rho \bar{v}_{\psi}
$$

Using (24) the stream function and the nominally streamwise component of vorticity, $\Omega_{3}$, are related by

$$
\begin{aligned}
\Omega_{3} & =\nabla_{S} \times \bar{v}_{S} \\
& =\nabla_{S} \times\left(\bar{v}_{\phi}+\bar{v}_{\psi}\right) \\
& =\nabla_{S} \times \bar{v}_{\psi} \\
\Omega_{3} & =\nabla_{S} \times\left(\frac{1}{\rho} \nabla_{S} \times \bar{\psi}\right)
\end{aligned}
$$

Equations (20) and (26) are solved simultaneously for $\bar{\psi}$ and $s_{3}$. Since the remaining parameters are either known functions or expressible in terms of known functions, $\Omega_{3}$ and $\bar{\psi}$.

Flow in Ducts with Superelliptic Cross Sections

The analysis of the previous section was applied to the flow in a general class of subsonic diffuser ducts. The duct centerline is defined by polynomials and the cross sections are described by superellipses in a plane locally normal to the centerline. The duct boundaries are coordinate surfaces, greatly simplifying the boundary conditions. This coordinate system is described in detail in Ref. 2 . Note that the grid distribution functions of Ref. 2 have been replaced. For radial distributions to resolve the boundary layer: 


$$
R=\frac{\tanh (D r)}{\tanh (D)}
$$

where $R$ is physical radius, $r$ is the radial computational coordinate parameter and $D$, the damping function which determines the severity of the distribution, is controlled through the parameter $\alpha$ by:

$$
D=\frac{2}{1-a}
$$

A circumferential distribution function is also used to cluster grid points into regions of tight wall curvatures. The equally spaced computational coordinate $t$ is related to the physical angle $\theta$ in a quadrant by the relation:

$$
\begin{aligned}
& \Theta=A \sinh \left(\frac{D(t-C)}{t_{2}-t_{1}}\right)+B \\
& \dagger_{1}=0, \dagger_{2}=1 \\
& \Theta_{1}=0 \Theta_{2}=\pi / 2 \\
& \Theta_{C}=\tan ^{-1}\left(S^{2}\right) \\
& S=\operatorname{shape}(\text { ratio of major to minor axis }) \\
& E=\operatorname{superel1iptic~exponent} \\
& R=\frac{\Theta_{1}-\Theta_{C}}{\Theta_{2}-\Theta_{C}}, D=\ln (2 * E+2 * S) \\
& D Z M=\frac{\ln (2 * E+2 * S)}{\dagger_{2}-t_{1}}
\end{aligned}
$$

where:

$$
\begin{gathered}
C=\frac{1}{D Z M} \tanh ^{-1}\left[\frac{\sinh \left(D Z M \cdot t_{1}\right)-R \cdot \sinh \left(D Z M \cdot t_{2}\right)}{\cosh \left(D Z M \cdot t_{1}\right)-R \cdot \cosh \left(D Z M \cdot t_{2}\right)}\right] \\
B=\Theta_{1}+\frac{\Theta_{2}-\Theta_{1}}{1-\frac{\sinh \left(D Z M \cdot\left(t_{2}-C\right)\right)}{\sinh \left(D Z M \cdot\left(t_{1}-C\right)\right)}}
\end{gathered}
$$




$$
A=\frac{\Theta_{2}-\Theta_{1}}{\sinh \left(\operatorname{DZM}\left(t_{2}-C\right)\right)-\sinh \left(\operatorname{DZM}\left(t_{1}-C\right)\right)}
$$

This distribution function, (29), clusters grid points toward $\theta_{c} \theta_{c} \cdot$ Several examples of the effects of (27) and (29) are presented in Fig. 1 for a series of cross sectional shapes.

The governing equations used in this application are:

MOMENTUM in the $\mathrm{x}^{3}$ direction

$$
\begin{aligned}
\rho u_{j} \frac{\partial Y^{i}}{\partial X^{j}} \frac{\partial u_{3}}{\partial Y^{i}}= & -\frac{\partial Y^{i}}{\partial X^{3}} \frac{\partial P_{3}-D}{\partial Y^{i}}-\frac{\partial Y^{3}}{\partial X^{3}} \frac{\partial P_{1-D}}{\partial Y^{3}} \\
& +\frac{\partial Y^{i}}{\partial X^{j}} \frac{\partial}{\partial Y^{i}}\left[\mu _ { e } \left(\left(1-\delta_{j} 3\right)\left(\frac{\partial Y^{n}}{\partial X^{j}} \frac{\partial u_{3}}{\partial Y^{n}}+\frac{\partial Y^{n}}{\partial X^{3}} \frac{\partial u j}{\partial Y^{n}}\right)\right.\right. \\
& \left.\left.-\frac{2}{3} \delta_{3 j}\left(1-\delta_{k^{3}}\right) \frac{\partial Y^{n}}{\partial X^{k}} \frac{\partial u_{k}}{\partial Y^{n}}\right)\right]
\end{aligned}
$$

from CONTINUITY

$$
\begin{aligned}
\frac{\partial Y^{j}}{\partial X^{i}} \frac{\partial}{\partial Y^{j}}\left(\rho \frac{\partial Y^{k}}{\partial X^{i}}\right) & \frac{\partial \phi}{\partial Y^{k}}+\rho\left(\frac{\partial Y^{k}}{\partial X^{i}}\right)^{2} \frac{\partial^{2} \phi}{\partial Y^{k^{2}}} \\
& +2 \rho \frac{\partial Y^{\prime}}{\partial X^{i}} \frac{\partial Y^{2}}{\partial X^{i}} \frac{\partial^{2} \phi}{\partial Y^{\prime} \partial Y^{2}}=-\frac{\partial Y^{n}}{\partial X^{3}} \frac{\partial \rho u_{3}}{\partial Y^{n}}
\end{aligned}
$$

VORTICITY TRANSPORT in the $\mathrm{x}^{3}$ direction

$$
u_{j} \frac{\partial \Omega_{3}}{\partial x^{j}}-\Omega_{j} \frac{\partial u_{3}}{\partial x^{j}}=\frac{\mu_{e}}{\rho} \frac{\partial^{2} \Omega_{3}}{\partial x^{j 2}}
$$

STREAM FUNCTION

$$
\begin{aligned}
\frac{\partial Y^{j}}{\partial X^{i}} \frac{\partial}{\partial Y^{i}}\left(\frac{1}{\rho} \frac{\partial Y^{k}}{\partial X^{i}}\right) & \frac{\partial \psi}{\partial Y^{k}} \\
+ & \frac{1}{\rho}\left(\frac{\partial Y^{k}}{\partial X^{i}}\right)^{2} \frac{\partial^{2} \psi}{\partial Y^{k^{2}}} \\
& +\frac{2}{\rho} \frac{\partial Y^{i}}{\partial X^{i}} \frac{\partial Y^{2}}{\partial X^{i}} \frac{\partial^{2} \psi}{\partial Y^{\prime} \partial Y^{2}}=-\Omega_{3}
\end{aligned}
$$


The vorticity transport equation was used in simplified form for convenience during the development phase of this study. Use of equation (20) for vorticity transport is anticipated.

At each marching step the computational domain has two symmetry boundaries, a boundary which is the duct wall and a boundary which is a small tube surrounding the centerline singularity (see Fi. 2). The boundary conditions for the $\mathrm{x}^{3}$ momentum cquation are $\partial u / \partial n=$ ? on the two symmetry surfaces where $n$ is the local surface normal, $u=0$ on the wall and

at the centerline.

$$
\mathrm{u} \frac{d \mathrm{u}}{d \mathrm{x}^{3}}=-\frac{1}{\rho} \frac{d \mathrm{P}}{d \mathrm{x}^{3}}
$$

The scalar potential equation for $\phi$ has the boundary condition $\phi=0$ on all boundaries.

The vorticity and stream function equations are solved sequentially and coupled iteratively. The stream function boundary condition $\psi=0$ is applied at all boundarics. The vorticity boundary condition $\Omega_{3}=0$ is applied along the symmetry and centerline boundaries. On the wall the vorticity boundary condition is derived following Briley \& McDonald (Ref. 1), by writing the stream function equation (36) on the wall using central difference formulas. The additional constraint needed to eliminate the added variable is the no-slip condition for the cross flow. These equations are

$$
\begin{aligned}
& \frac{\partial Y^{j}}{\partial X^{i}} \frac{\partial}{\partial Y^{j}}\left(\frac{1}{\rho} \frac{\partial Y^{2}}{\partial X^{i}}\right) \frac{\partial \psi}{\partial Y^{2}}+\frac{1}{\rho}\left(\frac{\partial Y^{2}}{\partial X^{i}}\right)^{2} \frac{\partial^{2} \psi}{\partial Y^{2}} \\
& +\frac{2}{\rho} \frac{\partial Y^{1}}{\partial X^{i}} \frac{\partial Y^{2}}{\partial X^{i}} \frac{\partial^{2} \psi}{\partial Y^{i} \partial Y^{2}}=-s i_{3}
\end{aligned}
$$

and setting the slip velocity from the solution of the stream function-vorticity equations equal and opposite to the slip velocity from the solution of the scalar potential equation vields:

$$
\frac{\partial \psi}{\partial Y^{2}}=\rho \frac{\frac{\partial Y^{\prime}}{\partial X^{\prime}} V_{\phi}+\frac{\partial Y^{\prime}}{\partial X^{2}} W_{\phi}}{\frac{\partial Y^{\prime}}{\partial X^{2}} \frac{\partial Y^{2}}{\partial X^{\prime}}-\frac{\partial Y^{\prime}}{\partial X^{\prime}} \frac{\partial Y^{2}}{\partial X^{2}}}
$$


where

$$
v_{\phi}=\frac{\partial Y^{2}}{\partial X^{1}} \frac{\partial \phi}{\partial Y^{2}} \quad w_{\phi}=\frac{\partial Y^{2}}{\partial X^{2}} \frac{\partial \phi}{\partial Y^{2}}
$$

on the wall.

The mixing length model used in this analysis employs the eddy-viscosity formulation for the Reynolds stresses, i.e.,

$$
\rho \overline{v^{i} v i}=-\frac{\mu_{T}}{\operatorname{Re}} \frac{\partial v^{j}}{\partial x_{i}}
$$

Hence, this formulation still suffers from the physical shortcoming that there is zero Reynolds stress wherever the velocity gradient is zero. In addition, the eddy viscosity formulation is isotropic which may be incorrect in many three-dimensional and swirling flows. However, for practical calculations of turbulent internal flows there are as yet no other transport models which are either suitable or as relatively well developed.

The mixing length turbulence model employed in this analysis is based on a mixing length distribution. The mathematical form of the expression for the turbulent viscosity follows from Ref. 3:

$$
\frac{\mu_{T}}{\operatorname{Re}}=\rho \ell^{2}(2 \overline{\bar{e}}: \overline{\bar{e}})^{1 / 2}
$$

where $\overline{\bar{e}}$ is the mean flow rate of strain tensor

$$
\overline{\bar{e}}=1 / 2\left[(\nabla \vec{v})+(\nabla \vec{v})^{T}\right]
$$

The mixing length $\ell$ is determined from the Buleev formula (Ref. 4)

$$
\frac{1}{\ell}=\frac{1}{2 \kappa} \int^{2 \pi} \frac{1}{S} d \theta
$$

where $K$ is the von Karman constant and $S$ and $\Theta$ are defined in Fig. 3.

\section{Potential Flow}

The present application of the analysis presented in the previous section uses a three-dimensional potential flow to supply the a priori known pressure field used in the viscous flow analysis. The potential flow is computed in the computational coordinate system by solving the equation

$$
\begin{gathered}
\nabla^{2} \phi=0 \\
\frac{\partial^{2} \phi}{\partial x^{1^{2}}}+\frac{\partial^{2} \phi}{\partial x^{2^{2}}}+\frac{\partial^{2} \phi}{\partial x^{3^{2}}}=0
\end{gathered}
$$

or expressed in the computational coordinate system: 


$$
\begin{aligned}
\frac{\partial Y^{i}}{\partial X^{1}} \frac{\partial}{\partial Y^{i}}\left(\frac{\partial Y^{j}}{\partial X^{1}} \frac{\partial \phi}{\partial Y^{j}}\right) & +\frac{\partial Y^{i}}{\partial X^{2}} \frac{\partial}{\partial Y^{i}}\left(\frac{\partial Y^{j}}{\partial X^{2}} \frac{\partial \phi}{\partial Y^{j}}\right) \\
& +\frac{\partial Y^{i}}{\partial X^{3}} \frac{\partial}{\partial Y^{i}}\left(\frac{\partial Y^{j}}{\partial X^{3}} \frac{\partial \phi}{\partial Y^{j}}\right)=0
\end{aligned}
$$

where $\phi$ is the velocity potential, $x^{i}$ are the Cartesian coordinates and $y^{i}$ are the computational coordinates. This equation is solved using an iterative scalar ADI procedure. The boundary conditions at the duct wall is that there is no flow through the wall, i.e. $\frac{\partial \phi}{\partial n}=0$. Since the potential, $\phi$, is dimensionless the inflow and outflow values are set to 0 and 1 respectively. The duct centerline would represent a coordinate singularity so the coordinate system used extends from the duct walls down to a small tube of finite radius around the centerline. The boundary condition applied at the centerline is derived from a zero velocity normal to the symmetry plane and permitting a velocity component tangent to the symmetry plane. Since the solution technique is iterative, the velocity boundary condition is lagged and updated every few iterations. The area in the tube around the centerline is small and this explicit, rather than implicit, handling of the boundary condition appears to have little if any adverse influence on the observed convergence properties of the solution procedure for solving Eq. (44).

Once the converged value of the potential, $\phi$, is found, the non-dimensional velocities at each grid point are computed. These velocities are scaled to be compatible with the specified reference conditions. From the velocities the corresponding pressure field is computed and values of pressure coefficient are computed and saved on permanent storage for later use in the viscous spatial marching calculations.

\section{Calculations}

The above analysis was applied to a series of lest problems. The first case presented here is the flow in the entrance region of a straight circular pipe. The calculations were compared to the experimental data of Reshotko (Ref. 5). The excellent agreement with the data is shown in Fig. 4 . 
The second case was of turbulent flow through a circular pipe undergoing an $\mathrm{S}$-shaped bend. The bend was offset 1 diameter in a streamwise distance of 5 diameters (Fig. 5). The entrance Mach number was 0.2 and the entrance Reynolds number was $10^{6}$. Although no test data was available to quantitatively evaluate the results, the test case was apparently successful. A qualitative evaluation has been made by comparing salient features of the computed flow with the flow measured in a model of the Boeing 727 Center Duct Inlet (Ref. 6). It must be emphasized that the computed case was for different geometry, Reynolds number and Mach number than the tested case. However, not only are the classical secondary flow patterns clearly discernible in both cases, but the development of the secondary flow patterns appears to accur in the same manner. The locations marked $A, B, C$ and $D$ refer to cross sections where comparisons will be presented. Sections A are near the center of the Eirst bend, sections $B$ are just after the inflection, sections $\mathrm{C}$ are just after the center of the second bend, and sections $D$ are at the ends of the ducts. Figure 6 presents the contours of total pressure at the bottom of the 727 duct for stations $A, B$ and $C$. The growing region of low total pressure is clearly seen. Also shown are contours of streamwise velocity at the bottom of the computed S-shaped duct. In the case presented these streamwise velocity contours have nearly the same shape as total pressure contours. Here again the growing region of low total pressure is clearly seen at the bottom of the duct. A comparison of sections $D$ in Figure 7 shows the classical total pressure contour shapes for S-shaped bends. Figure 8 also shows these classical exit plane total pressure contours from three other S-shaped bends (Ref. 7). 


\section{SUMMARY AND CONCLUSIONS}

An analysis is presented for computing three-dimensional subsonic flow in diffusers. Following Briley and McDonald, approximations are introduced into the Navier-Stokes equations to produce a system of equations which can be solved by forward marching. This paper reports on a study to adapt the above analysis for use with nonorthogonal constructed coordinate systems.

At each computational plane a local Cartesian coordinate system is defined at the duct centerline with one coordinate direction tangent to the centerline and another coordinate direction aligned with the outward facing normal. Special treatment of cases where the centerline curvature is zero is readily provided. Velocity components are defined to be aligned with these Cartesian directions and those governing equations that are vector equations are written in these local Cartesian directions. At each marching station the vector quantities computed at the previous station are transformed into these new vector directions.

The resulting partial differential equations are transformed into the constructed coordinate system. These equations contain only first derivatives of the elements of the transformation from Cartesian to constructed coordinates as contrasted with the familiar general orthogonal equations which contain second derivatives of the metric scale factors (h's). This reduced sensitivity of the governing equations to the details of the coordinate system permits more flexibility in the coordinate construction process. A family of duct shapes with superelliptic cross sections is currently available within the code representing a large class of diffuser shapes. These coordinates are nonorthogonal. The analysis developed in this study is applicable to more general cross-sectional shapes than are currently available within the code.

The above analysis was applied to subsonic viscous flow in several ducts. Qualitative agreement with experimental measurements was encouraging since the formation and migration of the vortex seen in the measurements was also present in the computed results. Additional experimental data is needed to provide a basis for detailed quantitative evaluation of the analysis.

The current version of the computer code solves the streamwise vorticity and stream function equations by coupling them through a sequential iterative procedure. A preferred method would be to solve these equations as an implicitly coupled block system. This is expected to improve convergence properties of these equations and permit larger streamwise steps to be taken. 


\section{REFERENCES}

1. Briley, W. R. and McDonald, H.: Analysis and Computation of Viscous Subsonic Primary and Secondary Flows, AIAA Paper No. 79-1453.

2. Eiseman, P. R., McDonald, H. and Briley, W. R.: Method for Computing ThreeDimensional Viscous Diffuser Flows. United Technologies Research Center Report R75-911737-1, July 1975.

3. Beer, J. M. and Chigier, N. A.: Combustion Aerodynamics. Wiley, New York, 1972.

4. Buleev, N. I.: Theoretical Model of the Mechanisms of Turbulent Exchange in Fluid Flow. Atomic Energy Research Establishment (U.K.) Translation 957, 1963.

5. Reshotko, E.: Experimental Study of the Stability of Pipe Flow, Pt. 1, Establishment of an Axially Symmetric Poiseuille Flow. Jet Propulsion Laboratory Progress Report No. 20-364, October 1958.

6. Kaldschmidt, G., Syltebo, B. E., and Ting, C. T.: "Design and Results of Low Speed Performance Confirmation Model Test of a 727 Airplane Center Duct Inlet for Refanned JT8D Engines:, The Boeing Company, 1974.

7. Bansod, P. and Bradshaw, P.: "The Flow in S-shaped Ducts", The Aeronautical Quarterly, Vol. XXIII, Part 2, May 1972. 

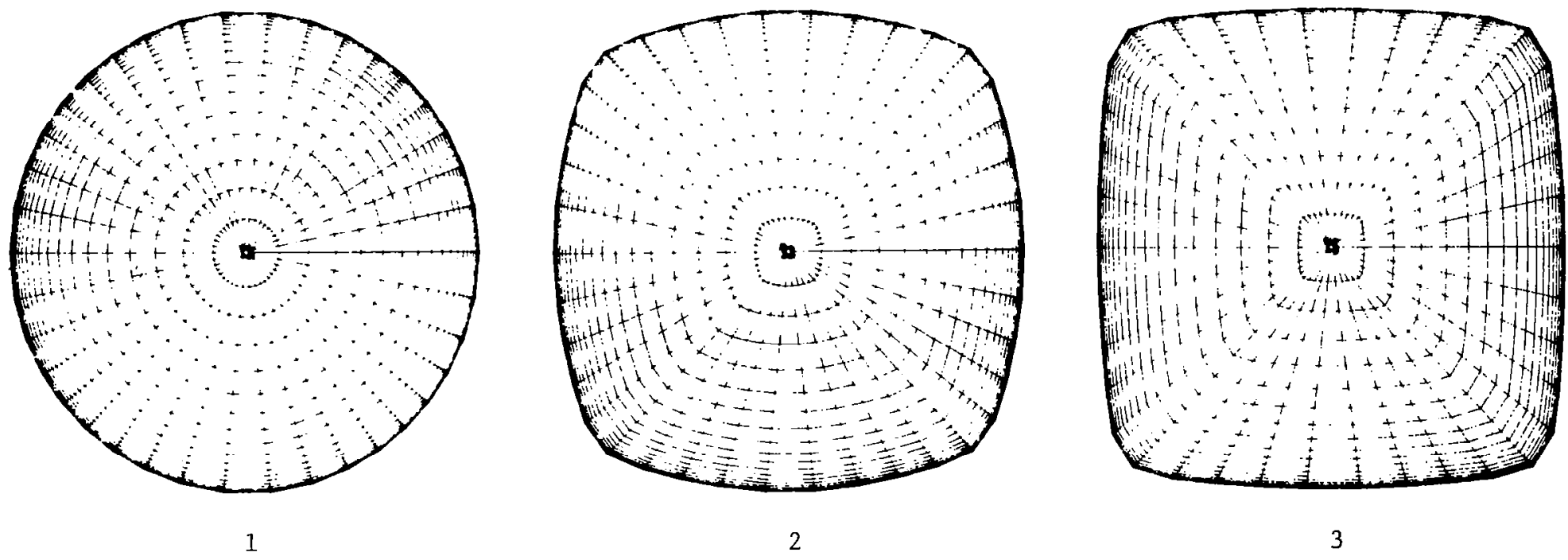

Fig. 1a - Variation in Superelliptic Exponent (Shape=1). 


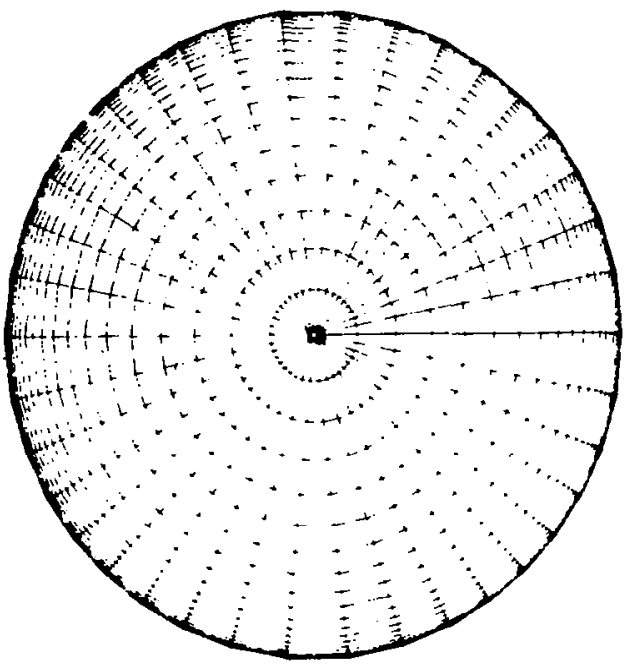

1

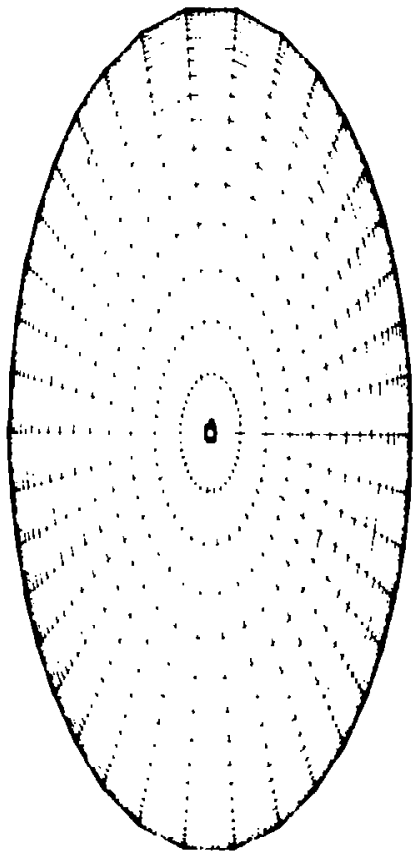

2

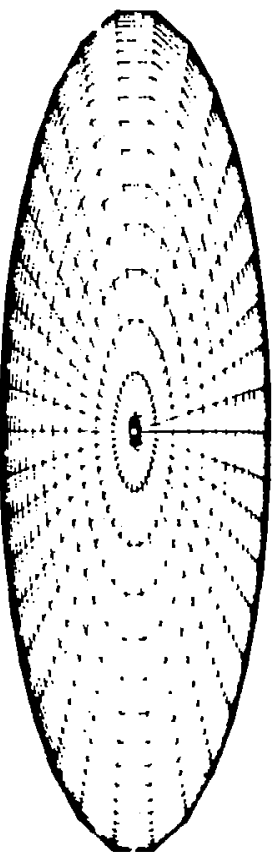

3

Fig. Ib - Variation in Shape (Exponent=2).

$\vdash$ 

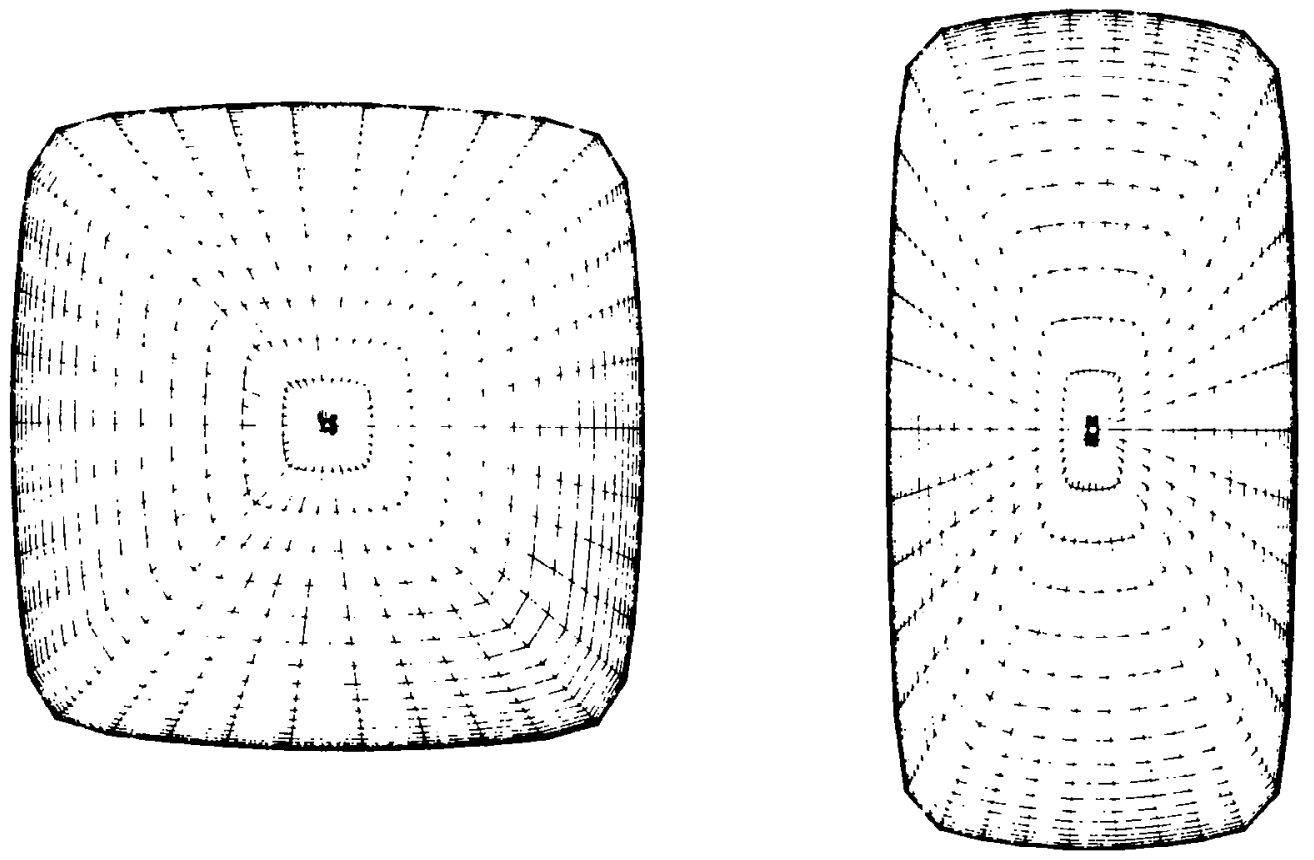

1

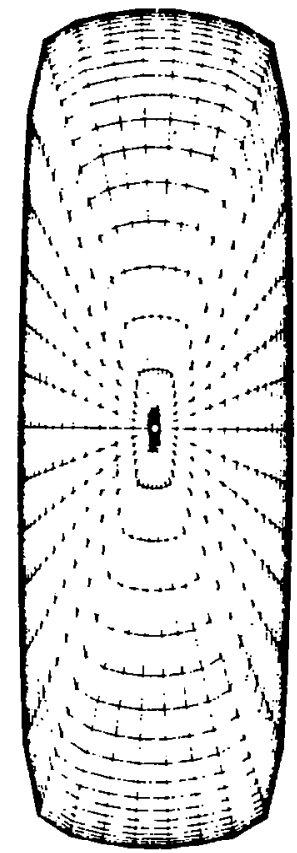

Fig. 1c - Variation in Shape (Exponent=5). 


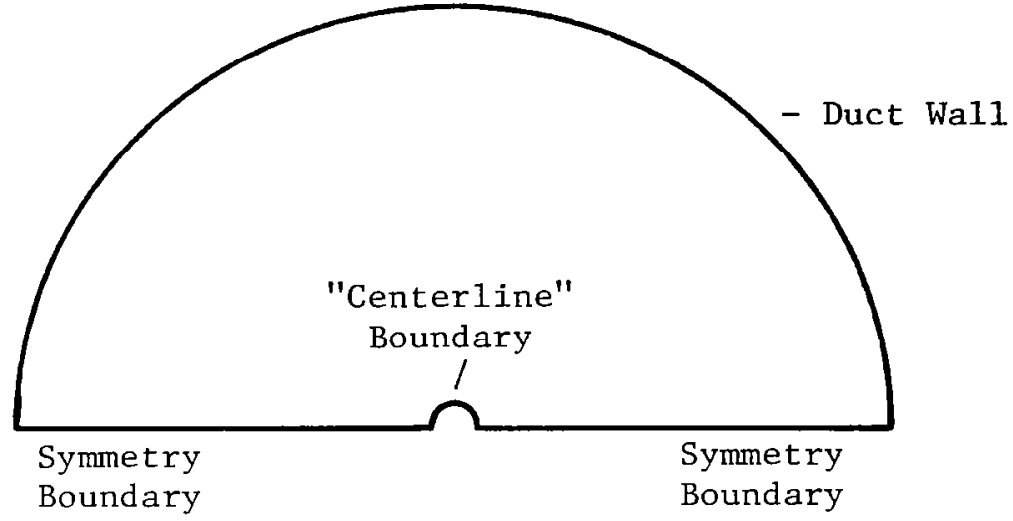

Fig. 2 - Computational Domain. 


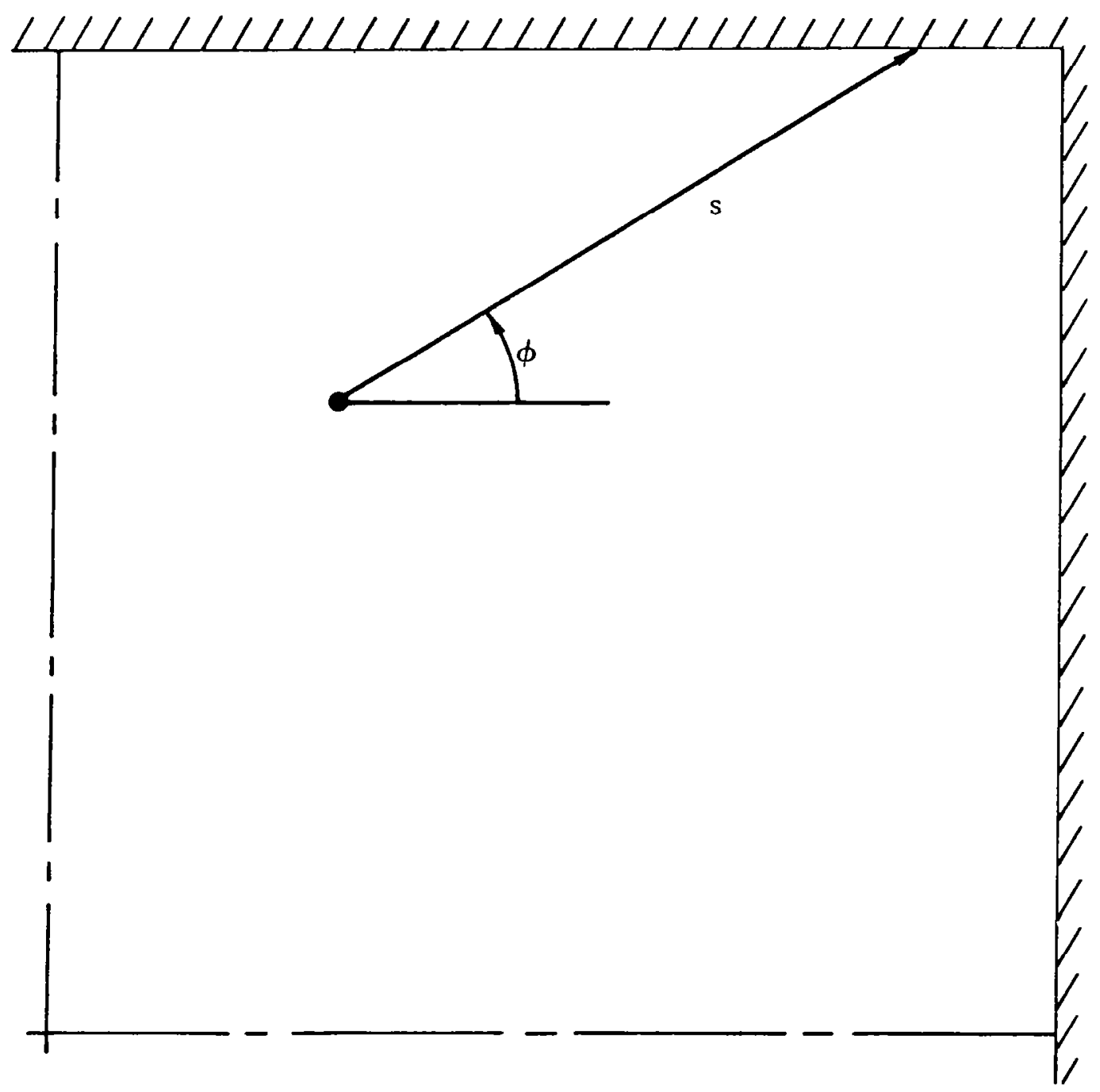

Fig. 3 - Notation for Calculating Buleev Length Scale. 
Current Prediction

$O \triangle$ Data of Reshotko (Ref.5)

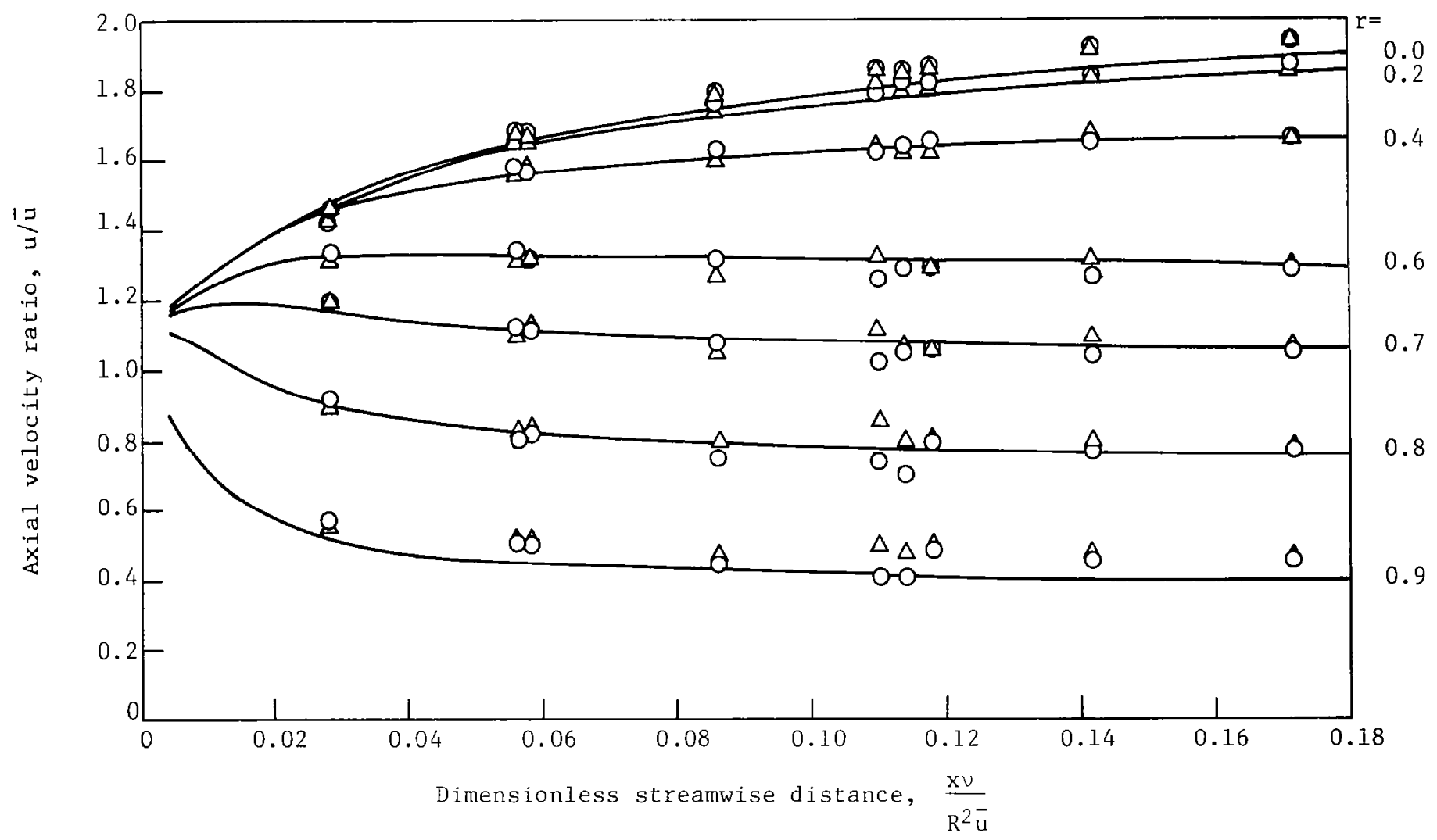

Figure 4. - Development of the velocity profile in a circular pipe 


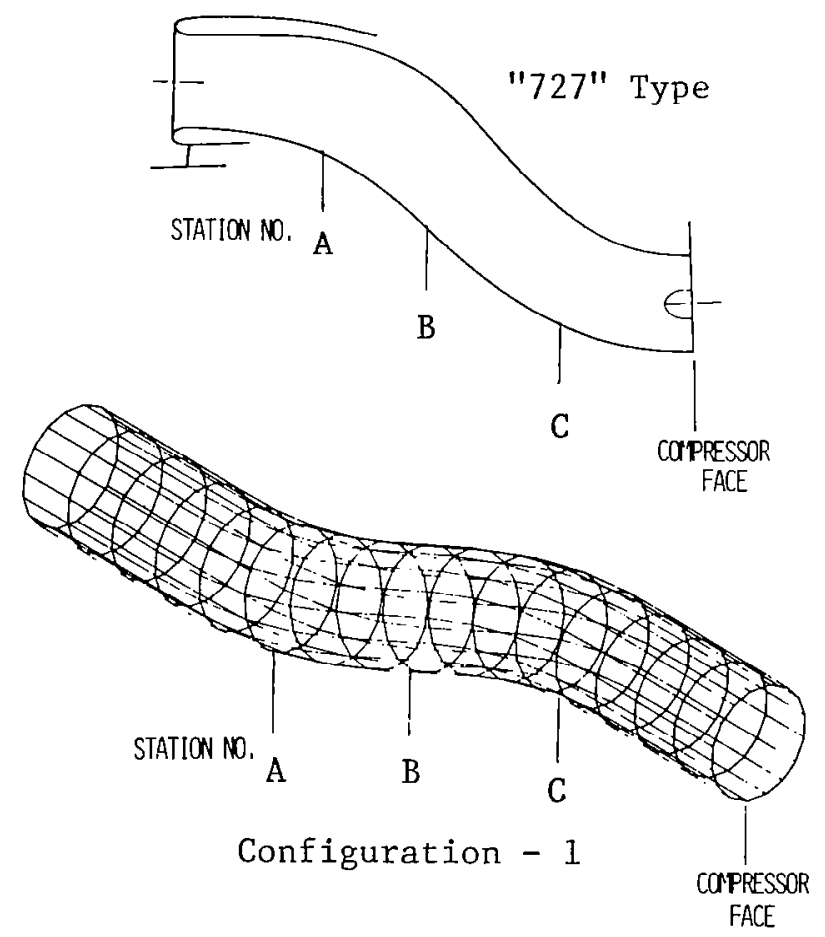

Fig. 5 - S-Duct Diffusers. 
"727"

Total Pressure
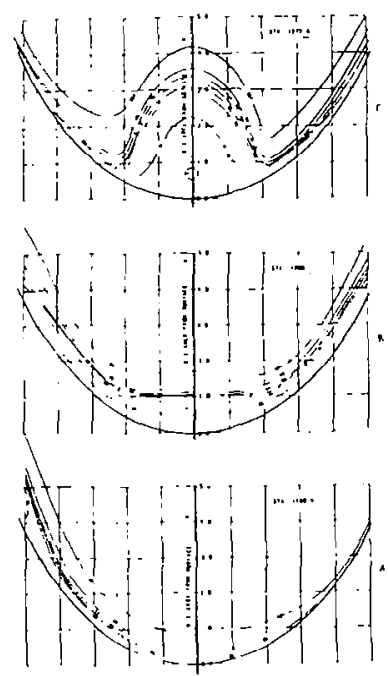

Configuration - 1

Total Velocity

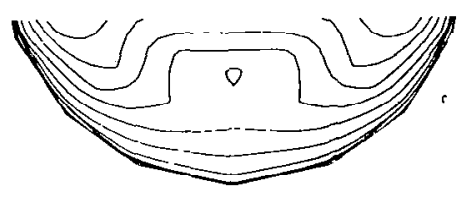

STATION C

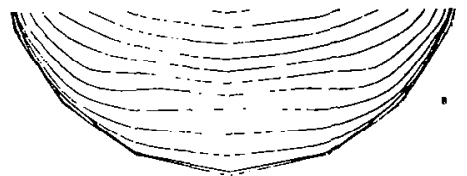

STATION B

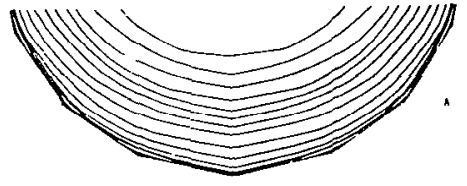

STATION A

Fig. 6 - Development of Deficit Region in S-Duct Diffusers. 
"727"

Total Pressure

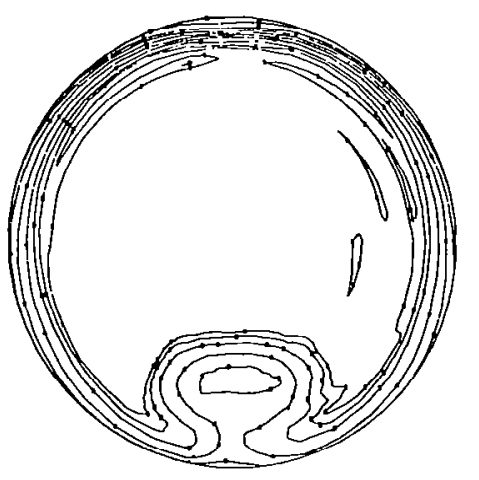

Configuration - 1

Total Velocity

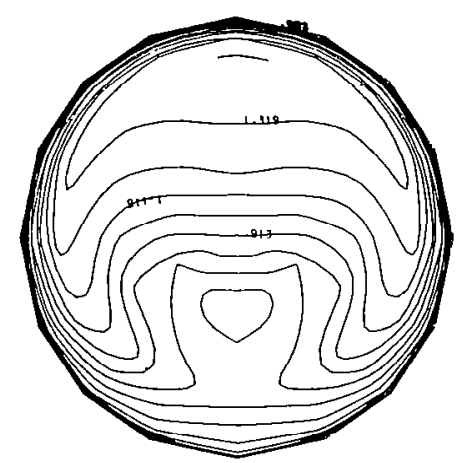

COPPEESOR FACE STATION

Fig. 7 - Compressor Face Profiles. 

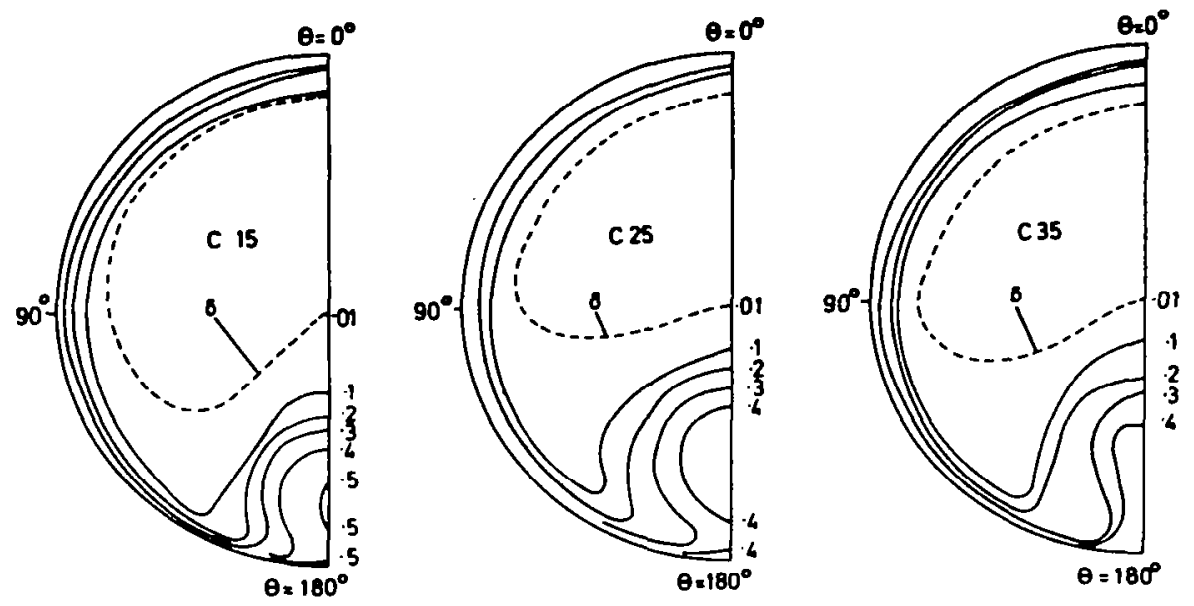

Figure 8. - Contours of total-pressure loss coefficient at exit, (Ref. 5). 
$x$

$y^{i}$

$\overline{\mathbf{i}}, \overline{\mathrm{j}}, \overline{\mathrm{k}}$

$\delta_{i j}$

$\overline{\mathrm{V}}$

$\mathrm{U}_{\mathrm{i}}$

$\mathrm{P}$

$\rho$

$\operatorname{Re}$

$\mu$

$\mu_{\mathrm{e}}$

$\Omega_{i}$

$\phi$

$\psi$

$\mathbf{r}$

$\mathrm{t}$
Cartesian coordinate

General curvelinear coordinate

Cartesian unit vectors

Kroniker delta

Velocity vector

Velocity component

Pressure

Density

Reynolds number

Viscosity

Effective turbulent viscosity

Vorticity component

Scalar potential function

Stream function

Radial computational coordinate parameter

Azimuthal computational coordinate parameter 


\section{APPENDIX \\ COMPUTER CODE INPUT AND OUTPUT}

The PEPSIG code input is of the form:

MODE, TITLE

Namelist Data

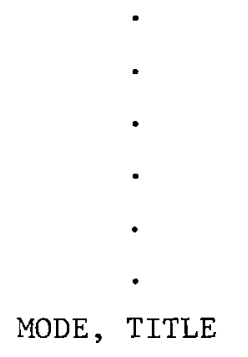

Namelist Data

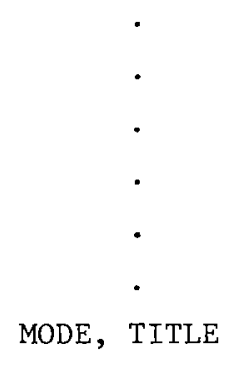

Name1ist Data

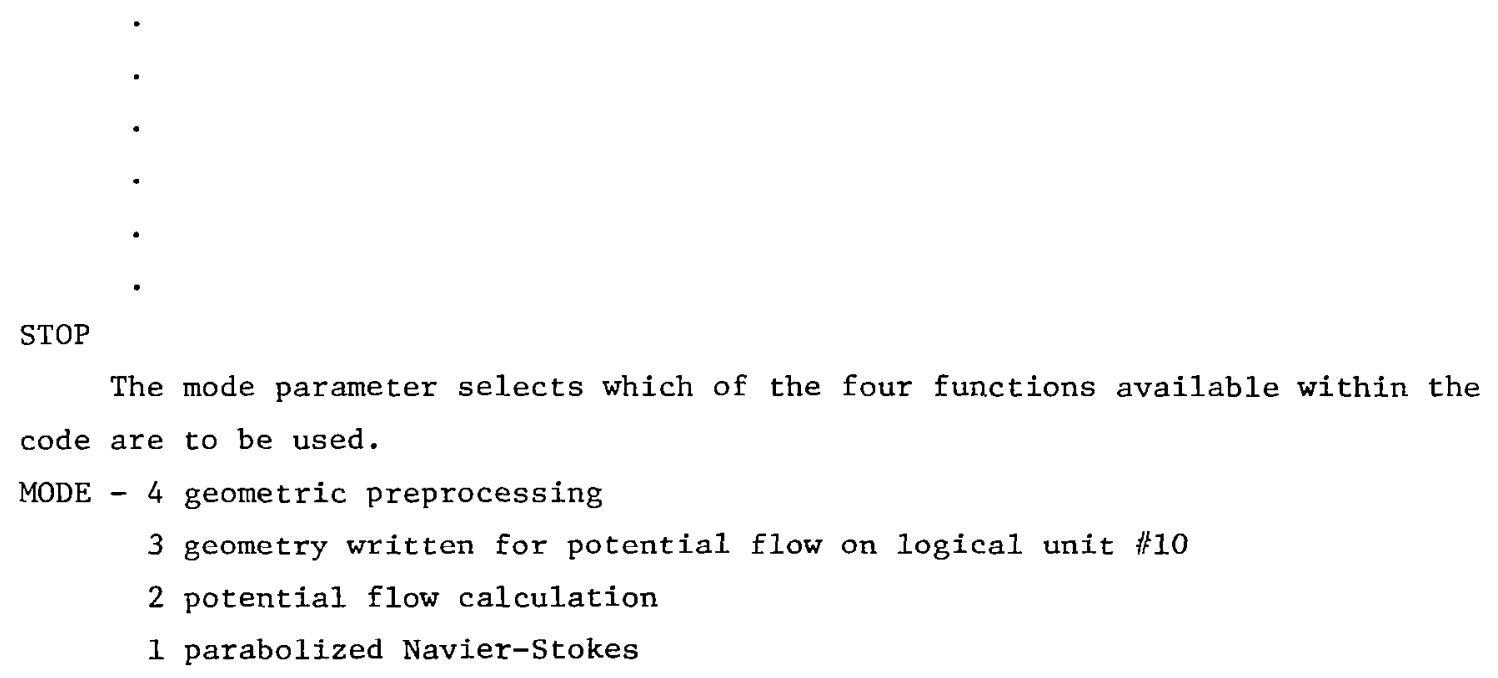


When operating in MODE $=4$, the code will solve a series of simultaneous equations to determine polynomials $Y=f(X)$ from tabulations of $Y, Y^{\prime}$ and/or $Y^{\prime \prime}$ versus $X$. The input is specified through NAMELIST:

$\underline{\$ L}$

NEQ - number of equations

XIN - input table of $X$ values

YIN - input of $Y, Y^{\prime}$ or $Y^{\prime \prime}$ according to corresponding value of ID

ID - O YIN is $\mathrm{Y}$

1 YIN is $Y^{\prime}$

2 YIN is $\mathrm{Y}^{\prime \prime}$

NX - number of locations in the interval $\mathrm{X} 1$, $\mathrm{X} 2$ at which the resulting polynomial is to be evaluated.

$\mathrm{X} 1, \mathrm{X} 2$ - interval in which the resulting polynomial is to be evaluated

The input for MODE $=3,2$ and 1 is provided through three Namelist tables. Although the code is still in the development stage and elegant input has not yet been devised, the Namelist method provides a serviceable means of setting up the cases to be run. The three Namelists are, in order of being read: GEOM, FLUIDS, and SWITCH. The numbers in parenthesis following the explanation of the input variable are the recommended values.

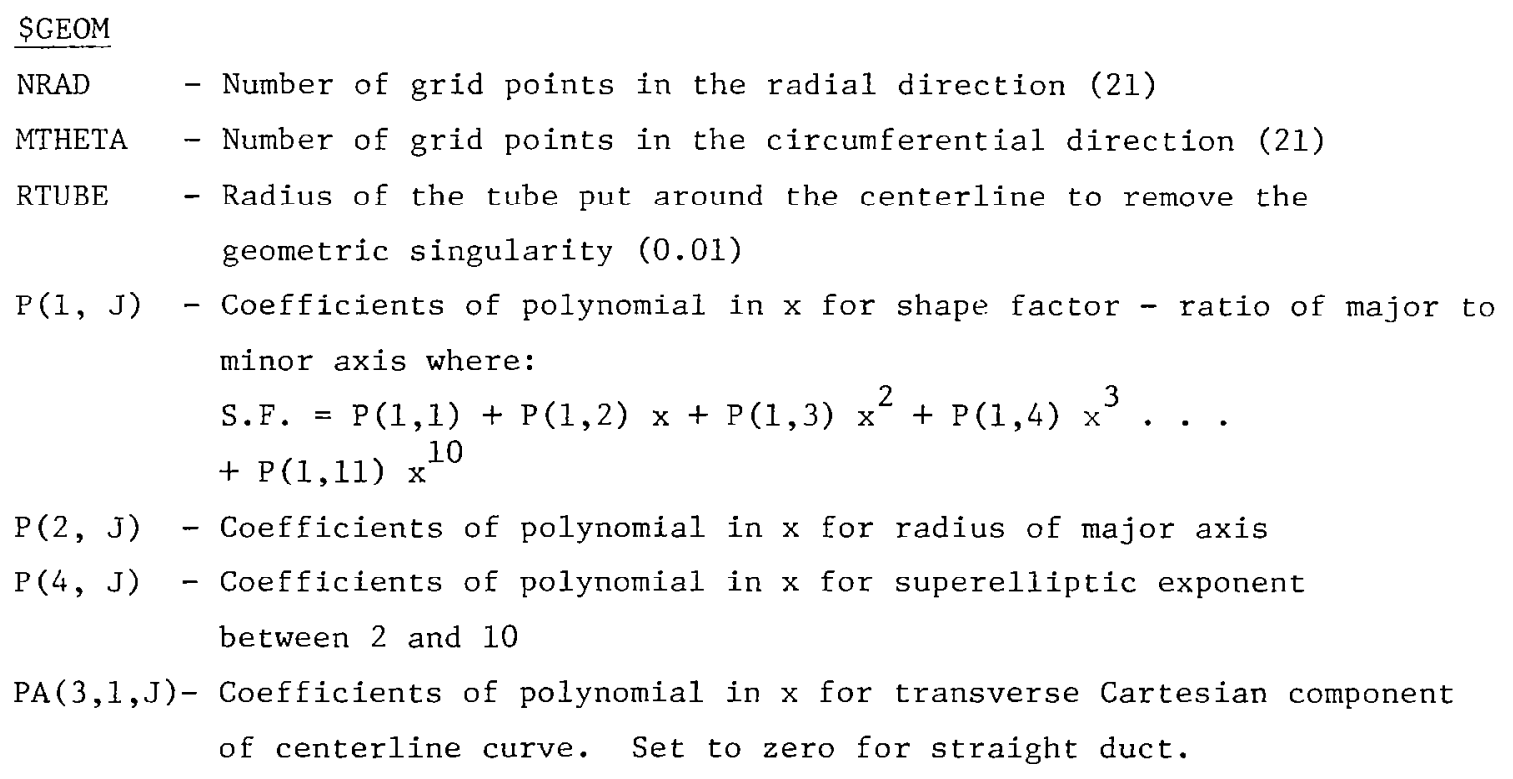


NOTE: The $P$ and PA arrays determine the location of the grid points. Since the position vector of the grid points is differentiated twice within the code, the piecewise polynomials describing these locations must be continuous to two derivatives. Small values of higher derivatives is also recommended.

YZERO - Reference length in $\mathrm{ft} .(1.0)$

NS - Number of the last marching station to be computed

NFIRST - Number of the first marching station whose geometry is to be specified (usually 1 )

$X(N F I R S T)$ - Nondimensionalized location of first marching step whose geometry is to be specified

DX - Initial step size

AP - Ratio of consecutive step sizes in the marching direction $x(i)=x(i-1)+A P *[x(i-1)-x(i-2)]$

IPA - Circumferential distribution function, 2 is equally spaced, 3 is automatic distribution, (3)

VIS $\quad-\alpha$ in Eq. (28) (.3)

IPB - Radial distribution function 2 is equally spaced, 3 is packed toward the wall (3)

IRSTIN - The station number to be read in for restart If IRSTIN $=0$, not a restart case

IRSTOT - The interval for saving restart information If $\operatorname{IRSTOT}=0$, no restart information saved

JRSTIN - Logical file name from which restart information is to be read

JRSTOT - Logical file name onto which restart information is to be written

NFILE - The sequence number in JRSTIN of the desired restart information

NSAVED - The number of restart stations saved on JRSTOT; must be initialized in inputs to the number of stations already written (and to be preserved) on JRSTOT. Nominally initialized NSAVED $=0$.

NOTE: By setting JRSTOT $=$ JRSTIN and NFILE = NSAVED, one file can be used for both read and write information, without destroying the already saved information. \$FLUIDS

RZERO - Reference density in $1 \mathrm{bm} / \mathrm{ft}^{3}$
UZERO


NOTE: Set either CMACH or UZERO and the other will be computed within the code.

REY - Reynolds number based on UZERO, YZERO and RZERO. Reference viscosity is determined by VISCOS = UZERO*YZERO+RZERO/REY

KTURB - 0 Laminar

- 1 Turbulent

YSLOT(2) - Initial nondimensionalized boundary layer thickness

\section{\$SWITCH}

NE1PF - Number of circumferential grid points used in potential flow

NE2PF - Number of radial grid points used in potential flow

IGDMP - 2 Dumps streamwise momentum equation coefficients loaded into matrix inverter

0 No dump

IPLOT - 1 Write plot file (logical unit \#8)

0 No plot file

$\operatorname{ICOEF}(1, N)$ - Auxiliary array which controls various options within the code.

In general a value of 0 means the option is off, a value of 1 means on.

$\mathrm{N}$

1 COEFG convective terms printout

2 COEFG first viscous terms printout

3 COEFG second viscous terms printout

4 COEFG inviscid pressure gradient printout

5 COEFG viscous pressure gradient printout

6 PFIELD, CPI $=0$

7 PFLOW inter dump -1 , full dump 1 or 2

8 EI dump from CROSEC

9 output PHI, PSI velocities

10 output PHI, PSI Cartesian velocities

11 output Cartesian velocities

12 ADI dump PSI

13 ADI dump vorticity

14 ADI dump PHI 
15 input NAMELIST dump

16 IPRFSE dump

17 DYFA mixed der. diagnostic message

18 level 2 output

19 PFIELD drum reading information

20 output for ITER=1,2

$\operatorname{ICOEF}(2, N) N$

1 wall vorticity for no slip (COEFVS)

2 No wall damping on NLEN

3 COEFVS iteration print

4 Buleev mixing length formula

5 number of pressure iterations in ADICUP

6 auxiliary variable printout

7 auxiliary variable printout

8 auxiliary variable printout

9 auxiliary variable printout

10 auxiliary variable printout

Il auxiliary variable printout

12 auxiliary variable printout

13 auxiliary variable printout

14 PFLOW intermediate solutions

15 FRAME printout

16 ADI convergence information

17 DPDXV iteration printout

18 printout 3 level velocity and vorticity

NOTE:

It is advisable when running the potential flow calculation for the elliptic pressure field (MODE=3,2) to add constant area extensions both upstream and downstream of the duct to be evaluated in order to minimize the effects of the upstream and downstream boundary conditions. 
The output for $M O D E=4$ presents the coefficients of the polynomial and an evaluation of the polynomial at $\mathrm{NX}$ points in the range from X1 to X2. Displayed are the values of the polynomial along with the first and second derivative.
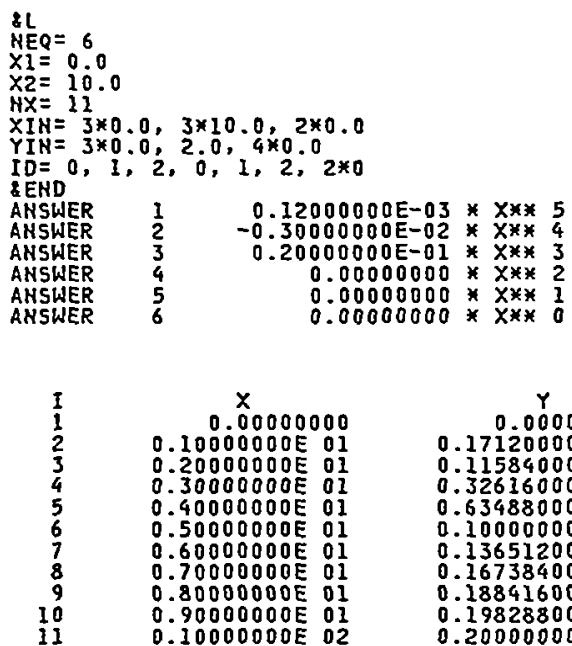

$Y$

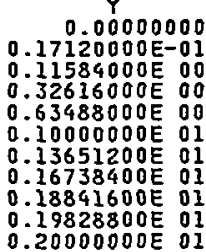

YP

0.00000000 0.48600000 E- 01 $0.15360000 \mathrm{E} 00$ 0.26460000 O 00 0.34560000 E 00 0.37500000 E 00 0.34560000 E 00 $0.15360000 \mathrm{E} 00$ $0.48600000 \mathrm{E}-01$

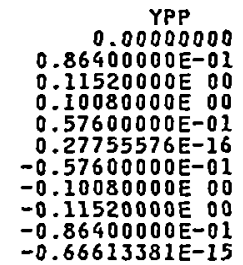

The output for $\mathrm{MODE}=3$ provides summary tables of geometric information including the absolute value of the radius of curvature of the centerline.

$x$

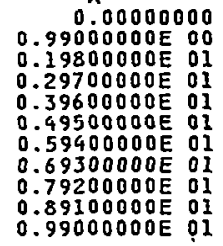

\section{$x$}

0.00000000 $0.99000000 E 00$ 0.19800000 E $0.29700000 \mathrm{E}$ OI .39600000 E 01 0.49500000 . 0.59400000 E 0.69300000 E 01 0.79200000 E 01 $0.89100000 E$
0.99000000 OI
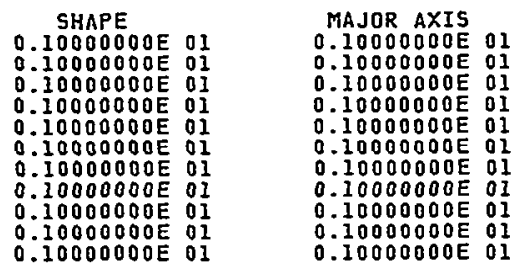

CENTERLINE LOCATION $(x 1, \times 2, x 3)$ $X 1$

0.00000000

$0.11279103 \mathrm{E}$ 00

$0.318267500_{00}$

$0.62110264 \mathrm{E} 00$

0.13442822 E

0.16550729 E

$0.18715030 \mathrm{E}$ o

$0.19781495 \mathrm{E}$ 01
ROTATION

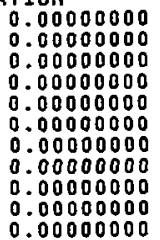

0.00000000

$x 3$

0.00000000

0.00000000

0.00000000

0.00000000

0.00000000

0.00000000

0.00000000

0.00000000

0.00000000

0.00000000
0.00000000
0.00000000 $0.99000000 E 00$ 0.19800000 E OI 0.29700000 E 01 0.39600000 E 01 0.49500000 E 01 0.59400000 OI 0.69300000 OI 0.79200000 E OI $0.89100000 E$ OI
EXPONENT $0.20000000 E$ OI 0.20000000 E (200000 . . 0.2000000 O 0.20000000 E ol 0.2000000 E 01 0.20000000 O

RAD OF CURVE 0.00000000 0.11688730 O2 $0.10856523 \mathrm{E}$ O2 $0.19796253 \mathrm{E} 02$ $0.40607325 \mathrm{E} 03$ 0.21837661 E 02 $0.11290609 \mathrm{E} \mathrm{O2}$ 0.90087385 E $0.11025311 \mathrm{E} 02$ 
Upon successful completion of writing the geometry on file \#10 the following message is written:

GEOMETRY WRITTEN FROM STATION 5 AT $X=0.9900 E$ DO TO STATION 14 AT $X=0.9900 E$ OI FOR ITCNT= 3

The output for $M O D E=2$ consists of intermediate printout information about the iteration followed by a tabulation of the information written on the pressure field file logical unit 13. Listed at each transverse computational plane are the streamwise plane number, the parametric location of the centerline, the radius from the centerline of each row of grid points expressed as a fraction of the local duct radius, the azimuthal location in radians of each radial line of grid points, and a two-dimensional display of pressure coefficient, $C_{p}$, at each node used in the potential flow calculation.
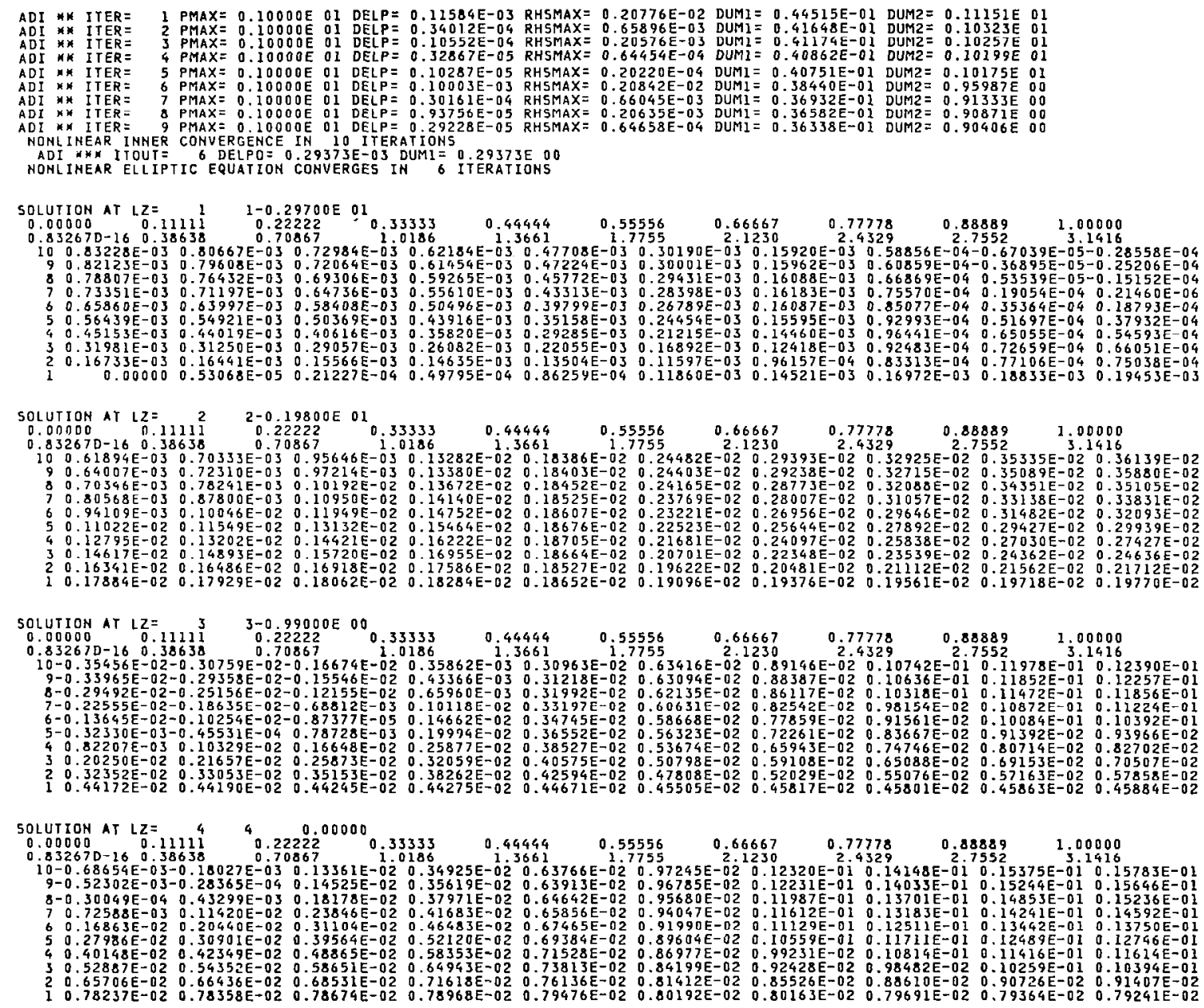
The output for MODE=l consists of the geometric summary information shown for MODE=3. Also presented at each streamwise station are the cross sectional area of the duct and the mass flux. Two-dimensional tabulations of several parameters are also provided in the cruss plane. These are:

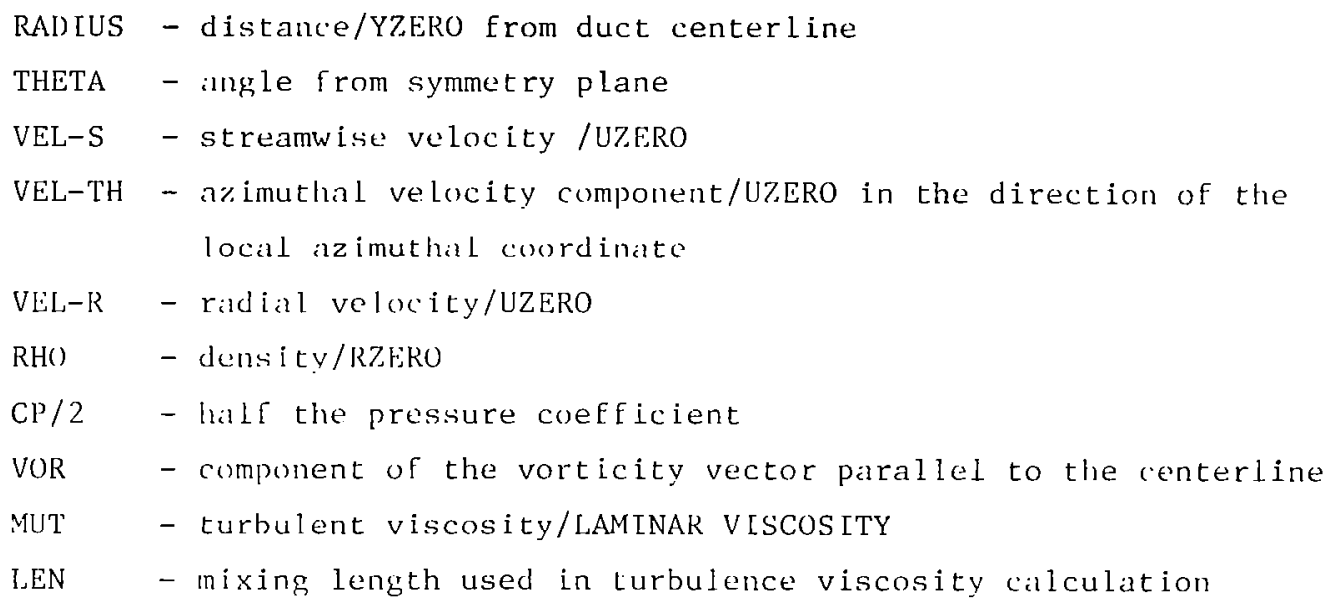




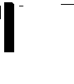

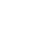

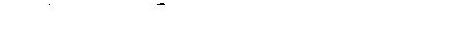

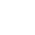

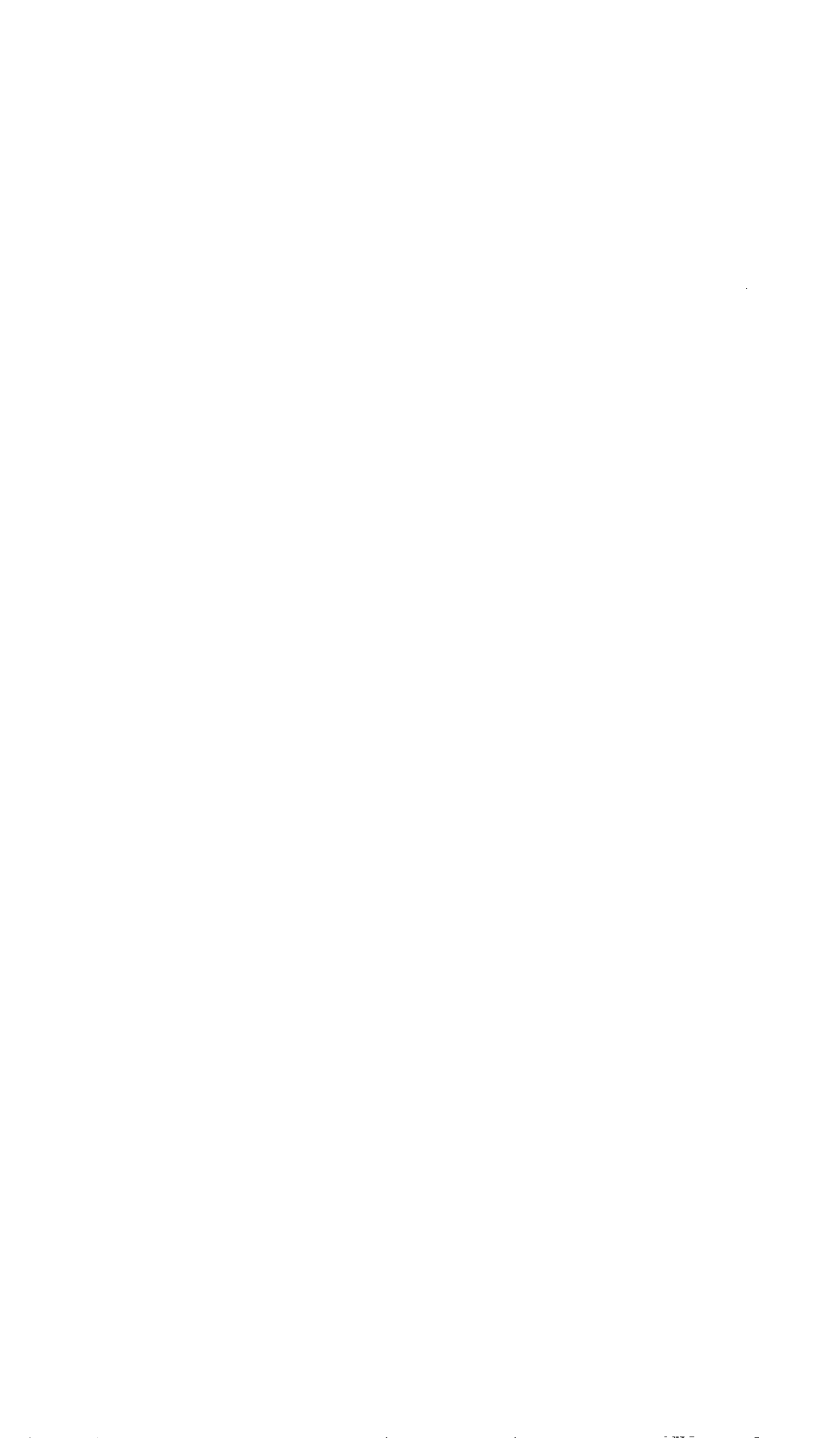




\begin{tabular}{|c|c|c|c|c|}
\hline $\begin{array}{l}\text { 1. Report No. } \\
\text { NASA CR-3389 }\end{array}$ & \multicolumn{2}{|c|}{ 2. Government Accession No. } & \multicolumn{2}{|c|}{ 3. Recipient's Catalog No. } \\
\hline \multirow{2}{*}{\multicolumn{3}{|c|}{$\begin{array}{l}\text { 4. Title and Subtitle A THREE-DIMENSIONAL TURBULENT COM- } \\
\text { PRESSIBLE SUBSONIC DUCT FLOW ANALYSIS FOR USE WITH } \\
\text { CONSTRUCTED COORDINATE SYSTEMS }\end{array}$}} & \multicolumn{2}{|l|}{$\begin{array}{l}\text { 5. Report Date } \\
\text { APRIL } 1981\end{array}$} \\
\hline & & & \multicolumn{2}{|c|}{ 6. Performing Organization Code } \\
\hline \multicolumn{3}{|c|}{$\begin{array}{l}\text { 7. Author(s) } \\
\text { R. Levy, H. McDonald, W. R. Briley, and J. P. Kreskovsky }\end{array}$} & \multicolumn{2}{|c|}{$\begin{array}{l}\text { 8. Performing Organization Report No. } \\
900005-F\end{array}$} \\
\hline \multirow{3}{*}{\multicolumn{3}{|c|}{$\begin{array}{l}\text { 9. Performing Organization Name and Address } \\
\text { Scientific Research Associates, Inc. } \\
\text { P.O. Box } 498 \\
\text { Glastonbury, CT } 06033\end{array}$}} & \multicolumn{2}{|l|}{ 10. Work Unit No. } \\
\hline & & & \multicolumn{2}{|c|}{$\begin{array}{l}\text { 11. Contract or Grant No. } \\
\text { NAS3-21735 }\end{array}$} \\
\hline & & & \multirow{2}{*}{\multicolumn{2}{|c|}{$\begin{array}{l}\text { 13. Type of Report and Period Covered } \\
\text { Contractor Report }\end{array}$}} \\
\hline \multirow{2}{*}{\multicolumn{3}{|c|}{$\begin{array}{l}\text { 12. Sponsoring Agency Name and Address } \\
\text { National Aeronautics and Space Administration } \\
\text { Washington, D.C. } 20546\end{array}$}} & & \\
\hline & & & \multicolumn{2}{|c|}{$\begin{array}{l}\text { 14. Sponsoring Agency Code } \\
505-04-12\end{array}$} \\
\hline \multicolumn{5}{|c|}{$\begin{array}{l}\text { 15. Supplementary Notes } \\
\text { Final report. Project Manager, Charles E. Towne, Propulsion Systems Division, NASA } \\
\text { Lewis Research Center, Cleveland, Ohio } 44135 .\end{array}$} \\
\hline \multicolumn{5}{|c|}{$\begin{array}{l}\text { 16. Abstract } \\
\text { An approximate analysis is presented for computation of three-dimensional subsonic flow in straight and } \\
\text { curved diffusers. The development parallels that of Briley and McDonald for forward-marching solution of } \\
\text { viscous primary and secondary flows, but differs in the coordinate formulation used and in details of the } \\
\text { approximations. The present formulation is intended to facilitate the use of constructed coordinates in cir- } \\
\text { cumstances where it is difficult to maintain smooth behavior in higher derivatives. This analysis is appli- } \\
\text { cable to nonorthogonal coordinate systems having a curved centerline and planar transverse coordinate sur- } \\
\text { faces normal to the centerline. The primary flow direction is taken to coincide with the local direction of the } \\
\text { duct centerline and is hence normal to transverse coordinate planes. The formulation utilizes vector compo- } \\
\text { nents (velocity, vorticity, transport equations) defined in terms of local Cartesian directions aligned with the } \\
\text { centerline tangent, although the governing equations themselves are expressed in general nonorthogonal coor- } \\
\text { dinates. For curved centerlines, these vector quantities are redefined in new local Cartesian directions at } \\
\text { each streamwise location. The use of local Cartesian variables and fluxes leads to governing equations } \\
\text { which require only first derivatives of the coordinate transformation, and this provides for the aforemen- } \\
\text { tioned ease in using constructed coordinates. The analysis is applied to a particular family of duct and dif- } \\
\text { fuser geometries having curved centerlines and superelliptic cross sections, using a nonorthogonal con- } \\
\text { structed coordinate system. Present computed results are compared with available experimental measure- } \\
\text { ments for different but related flow conditions. Qualitative agreement is observed with regard to the forma- } \\
\text { tion of secondary flows, migration of streamwise vortices, and distortion of the primary flow. Additional } \\
\text { experimental data is needed to provide a basis for detailed quantitative evaluation of the analysis and flow } \\
\text { predictions. }\end{array}$} \\
\hline \multicolumn{2}{|c|}{$\begin{array}{l}\text { 17. Key Words (Suggested by Author(s)) } \\
\text { Three-dimensional flow; Turbulent duct flow; } \\
\text { Parabolized Navier-Stokes; Secondary flow; } \\
\text { 3-D diffuser; Constructed coordinates }\end{array}$} & \multicolumn{3}{|c|}{$\begin{array}{l}\text { 18. Distribution Statement } \\
\text { Unclassified - unlimited } \\
\text { STAR Category } 34\end{array}$} \\
\hline $\begin{array}{l}\text { 19. Security Classif. (of this report) } \\
\text { Unclassified }\end{array}$ & 20. Security Classif & $\begin{array}{l}\text { of this page) } \\
\text { assified }\end{array}$ & $\begin{array}{l}\text { 21. No. of Pages } \\
39\end{array}$ & $\begin{array}{l}\text { 22. Price } \\
\mathrm{A03}\end{array}$ \\
\hline
\end{tabular}

* For sale by the National Technical Information Service, Springfield, Virginia 22161 